\title{
Is my system of ODEs $k$-cooperative?
}

\author{
Eyal Weiss and Michael Margaliot
}

Version Date: March 20, 2020

\begin{abstract}
A linear dynamical system is called positive if its flow maps the non-negative orthant to itself. More precisely, it maps the set of vectors with zero sign variations to itself. A linear dynamical system is called $k$-positive if its flow maps the set of vectors with up to $k-1$ sign variations to itself.

A nonlinear dynamical system is called $k$-cooperative if its variational system, which is a time-varying linear dynamical system, is $k$-positive. These systems have special asymptotic properties. For example, it was recently shown that strong 2cooperative systems satisfy a strong Poincaré-Bendixson property.

Positivity and $k$-positivity are easy to verify in terms of the sign-pattern of the matrix in the dynamics. However, these sign conditions are not invariant under a coordinate transformation. A natural question is to determine if a given $n$-dimensional system is $k$-positive up to a coordinate transformation. We study this problem for two special kinds of transformations: permutations and scaling by a signature matrix. For any $n \geq 4$ and $k \in\{2, \ldots, n-2\}$, we provide a graph-theoretical necessary and sufficient condition for $k$-positivity up to such coordinate transformations.
\end{abstract}

We describe an application of our results to a specific class of Lotka-Volterra systems.

\section{INTRODUCTION}

The state-variables in many mathematical models represent quantities that can never attain negative values. In Markov chains [10] the state-variables represent probabilities, in compartmental systems [19] and biological occupancy models [26], [6] they represent the density in each compartment, and so on.

The continuous-time linear system

$$
\dot{x}=A x
$$

is called positive if its flow maps the non-negative orthant to itself [8]. In other words, for any non-negative initial state all the state-variables remain non-negative for all time $t \geq 0$. Since the difference between trajectories is also a trajectory, this can be stated as the following partial ordering property: if $a, b$ are two initial conditions with $a \leq b$ (i.e., $a_{i} \leq b_{i}$ for all $i$ ) then $x(t, a) \leq x(t, b)$ for all $t \geq 0$. It is wellknown that (1) is positive if and only if (iff) $A$ is Metzler, that is, $a_{i j} \geq 0$ for any $i \neq j$.

Positive systems have important and special properties [17]. For example, a positive LTI that is asymptotically

The research of MM is supported in part by research grants from the Israel Science Foundation and the US-Israel Binational Science Foundation.

EW is with the Dept. of Computer Science, Bar-Ilan University, Ramat Gan, 5290002, Israel. MM (Corresponding Author) is with the School of Electrical Engineering, and the Sagol School of Neuroscience, Tel-Aviv University, Tel-Aviv 69978, Israel. E-mail: michaelmeeng.tau.ac.il stable always admits a diagonal quadratic Lyapunov function [18]. Diagonal stability implies that certain associated nonlinear systems have a well-ordered behavior [12], [5].

The linear system (1) is called $k$-positive if its flow maps the set of vectors with up to $k-1$ sign variations to itself. Thus, 1-positive systems are positive systems. Ref. [25] derived simple necessary and sufficient conditions guaranteeing $k$-positivity for any $k$. These turn out to be sign-pattern conditions on $A$. For example, (1) is 2-positive iff $A$ has the following sign pattern:

$$
\begin{aligned}
& a_{1 n} \leq 0, a_{n 1} \leq 0, \\
& a_{i j} \geq 0 \text { for all } i, j \text { with }|i-j|=1, \\
& a_{i j}=0 \text { for all } i, j \text { with } 1<|i-j|<n-1 .
\end{aligned}
$$

Note that such an $A$ is not necessarily Metzler, as $a_{1 n}, a_{n 1}$ can be negative.

Positivity has far reaching applications also to nonlinear systems. Consider the nonlinear system

$$
\dot{x}=f(x),
$$

and assume that its solutions evolve on a convex statespace $\Omega \subset \mathbb{R}^{n}$. Let $J(x):=\frac{\partial}{\partial x} f(x)$ denote the Jacobian of the vector field. The nonlinear system is called cooperative if its solutions satisfy the partial ordering property described above. Recall (see e.g. [3]) that if $a, b$ are two initial conditions, and $z(t):=x(t, a)-x(t, b)$ then

$$
\dot{z}(t)=M(t) z(t) \text {. }
$$

where

$$
M(t):=\int_{0}^{1} J(r x(t, a)+(1-r) x(t, b)) \mathrm{d} r .
$$

Eq. (3) describes how the variation between two solutions evolves in time. Thus, (2) is cooperative iff the LTV system (3) is positive for all $a, b \in \Omega$ and all $t \geq 0$.

The quasi-convergence theorem of Hirsch states that almost every bounded solution of a strongly cooperative system converges to the set of equilibria [23]. Cooperative systems have found numerous applications is neuroscience, systems biology, and chemistry. Indeed, in these fields it is often the case that the effect of one state-variable on another is either excitatory or inhibitory, making it relatively easy to verify that the Jacobian $J(x)$ is Metzler for all $x$. Angeli and Sontag [4] introduced an important generalization of cooperative systems (and more generally monotone systems) to control systems, and derived a small gain theorem for the interconnection of such systems.

The nonlinear system (2) is called $k$-cooperative if the 
variational system (3) is $k$-positive for all $a, b \in \Omega$ and all $t \geq$ 0 . For $k=1$ this reduces to a standard cooperative system. It was recently shown that strongly 2-cooperative systems satisfy a strong Poincaré-Bendixson property: if $x(t, a)$ is a bounded solution and its omega limit set $\omega(a)$ does not include an equilibrium then $\omega(a)$ is a periodic solution [25]. These results are closely related to the seminal work of Mallet-Paret and Smith on monotone cyclic feedback systems [15].

The conditions for $k$-positivity and $k$-cooperativity are not invariant under coordinate transformations. For example, if $\dot{x}=A x$ and $y:=T x$, with $T$ a nonsingular matrix, then $\dot{y}=T A T^{-1} y$, and $T A T^{-1}$ may be Metzler even if $A$ is not Metzler. For an LTI there is a well-known spectral condition guaranteeing that the flow maps a proper cone to itself [7], but this condition does nor carry over naturally to the system (3). Furthermore, the set of vectors with up to $k-1$ sign variations is not a proper cone. Nevertheless, if $J(x)$ satisfies some sign pattern condition for all $x \in \Omega$ then clearly this carries over to $M(t)$ in (4).

This raises the following question.

Problem 1: Given the dynamical system (2) and a set of nonsingular matrices $\mathbb{T} \subseteq \mathbb{R}^{n \times n}$, is there a matrix $T \in \mathbb{T}$ and an integer $k$ such that $T J(x) T^{-1}$ satisfies the sign condition for $k$-positivity for all $x \in \Omega$ ?

For the case of positive systems this question is wellknown. The motivation for addressing it is stated for example in [14]: "The essential point is the following: if a system can be shown to possess a partial ordering in some coordinate system, then all essential dynamical properties of partially ordered systems (to be described) will hold, regardless of reference frame".

Recall that a matrix $S \in \mathbb{R}^{n \times n}$ is called a signature matrix if $S$ is diagonal, and its diagonal elements are plus or minus 1. Smith [24] solved Problem 1 for the particular case of cooperativity (i.e. 1-coopertivity) and transformations by a signature matrix.

Here we address Problem 1 for two types of transformations: permutations and scaling by a signature matrix. For any $k \in\{2, \ldots, n-2\}$ we give a necessary and sufficient graph-theoretic condition for $k$-cooperativity up to such transformations.

The notion of $k$-positive systems has also been extended to discrete-time systems [13], [2], [1], but here we consider only the continuous-time case.

The remainder of this note is organized as follows. The next section reviews known definitions and results that will be used later on. Section III presents the main results, and Section IV describes an application to nonlinear LotkaVolterra systems.

\section{Preliminaries}

We first briefly review $k$-positive systems. For two integers $i \leq j$, we use $[i, j]$ to denote the set $\{i, i+1, \ldots, j\}$. The non-negative orthant in $\mathbb{R}^{n}$ is $\mathbb{R}_{+}^{n}:=\left\{x \in \mathbb{R}^{n} \mid x_{i} \geq\right.$ $0, i \in[1, n]\}$. For a matrix $A \in \mathbb{R}^{n \times m}, A^{\prime}$ denotes the transpose of $A$.

\section{A. k-positive systems}

For a vector $x \in \mathbb{R}^{n} \backslash\{0\}$, let $s^{-}(x)$ denote the number of sign variations in $x$ after deleting all the zero entries, with $s^{-}(0)$ defined as zero. For example, for $n=6$ and

$$
y=\left[\begin{array}{llllll}
1 & 0 & -2.5 & 0 & 0 & 3
\end{array}\right]^{\prime},
$$

$s^{-}(y)$ is the number of sign variations in the vector $\left[\begin{array}{lll}1 & -2.5 & 3\end{array}\right]^{\prime}$, so $s^{-}(y)=2$. Let $s^{+}(x)$ denote the maximal possible number of sign variations in $x$ after replacing every zero entry by either minus or plus one. For example, for $y$ in (5) $s^{+}(y)$ is the number of sign variations in the vector $\left[\begin{array}{llllll}1 & 1 & -2.5 & 1 & -1 & 3\end{array}\right]^{\prime}$, so $s^{+}(y)=4$. Clearly,

$$
0 \leq s^{-}(x) \leq s^{+}(x) \leq n-1 \text { for all } x \in \mathbb{R}^{n} .
$$

For any $k \in[1, n-1]$, let

$$
\begin{aligned}
& P_{-}^{k}:=\left\{x \in \mathbb{R}^{n} \mid s^{-}(x) \leq k-1\right\}, \\
& P_{+}^{k}:=\left\{x \in \mathbb{R}^{n} \mid s^{+}(x) \leq k-1\right\} .
\end{aligned}
$$

For example, $P_{-}^{1}=\mathbb{R}_{+}^{n} \cup\left(-\mathbb{R}_{+}^{n}\right)$ and $P_{+}^{1}=\operatorname{int}\left(P_{-}^{1}\right)$. More generally, it is not difficult to show that $P_{-}^{k}$ is closed, and that $P_{+}^{k}=\operatorname{int}\left(P_{-}^{k}\right)$ for all $k[1]$.

Fix a time interval $-\infty \leq t_{0}<t_{1} \leq \infty$. Consider the linear time-varying (LTV) system:

$$
\dot{x}(t)=A(t) x(t), \quad x\left(t_{0}\right)=x_{0},
$$

where $A(\cdot):\left(t_{0}, t_{1}\right) \rightarrow \mathbb{R}^{n \times n}$ is a locally (essentially) bounded measurable matrix function of $t$. Pick $k \in[1, n-1]$. The LTV (6) is called $k$-positive it its flow maps $P_{-}^{k}$ to itself, and strongly $k$-positive if its flow maps $P_{-}^{k} \backslash\{0\}$ to $P_{+}^{k}$.

We say that $A(t)$ admits a sign pattern if there exists a symbolic matrix $\bar{A} \in\{*,+,-, 0\}^{n \times n}$ such that: if $\bar{a}_{i j}=0$ then $a_{i j}(t)=0$ for almost all $t \in\left(t_{0}, t_{1}\right)$, and if $\bar{a}_{i j}=+$ $\left[\bar{a}_{i j}=-\right]$ then $a_{i j}(t) \geq 0\left[a_{i j}(t) \leq 0\right]$ for almost all $t \in$ $\left(t_{0}, t_{1}\right)$. Note that if $\bar{a}_{i j}=*$ then there is no constraint on $a_{i j}(t)$, i.e. * denotes a "don't care".

Definition 1: [25] Pick $n \geq 4$ and $k \in[2, n-2]$. Let $\bar{A}_{k}^{n} \in\{*,-, 0,+\}^{n \times n}$ denote the sign matrix with:

(I) $\bar{a}_{i i}=*$ for all $i$;

(II) if $k$ is even [odd] then $\bar{a}_{1 n}, \bar{a}_{n 1}=-\left[\bar{a}_{1 n}, \bar{a}_{n 1}=+\right]$;

(III) $\bar{a}_{i j}=+$ for all $i, j$ with $|i-j|=1$;

(IV) $\bar{a}_{i j}=0$ for all $i, j$ with $1<|i-j|<n-1$.

For example, $\bar{A}_{2}^{4}=\left[\begin{array}{cccc}* & + & 0 & - \\ + & * & + & 0 \\ 0 & + & * & + \\ - & 0 & + & *\end{array}\right]$. Note that for $k$ odd $\bar{A}_{k}^{n}$ is in particular Metzler, but for $k$ even $\bar{A}_{k}^{n}$ is not necessarily Metzler. Also, $\bar{A}_{2}^{n}=\bar{A}_{4}^{n}=\cdots=\bar{A}_{2 k}^{n}$ for all $k$ such that $2 k \leq n-2$, and $\bar{A}_{3}^{n}=\bar{A}_{5}^{n}=\cdots=\bar{A}_{2 k+1}^{n}$ for all $k$ such that $2 k+1 \leq n-2$. Note also that $A(t)$ satisfies the sign pattern of both $\bar{A}_{k}^{n}$ and $A_{k+1}^{n}$ iff $A(t)$ is tridiagonal with non-negative entries on the super- and sub-diagonals for almost all $t$. An LTV system that satisfies such a sign pattern is called a totally positive differential system [20]. Nonlinear systems whose Jacobians satisfy such a sign pattern have 
been analyzed by Smillie [21], Smith [22], and others (see the tutorial paper [16] for more details).

Theorem 1: [25] Pick $n \geq 4$ and $k \in[2, n-2]$. The system (6) is $k$-positive on $\left(t_{0}, t_{1}\right)$ iff $A(t)$ admits the sign structure $\bar{A}_{k}^{n}$ for almost all $\left(t_{0}, t_{1}\right)$.

This implies that we can classify $k$-positivity, with $k \in$ $[2, n-2]$ to just two cases: odd-positivity i.e. the flow of (6) maps $P^{k}$ to itself for all odd $k \in[2, n-2]$, and even-positivity i.e. the flow of 6 maps $P^{k}$ to itself for all even $k \in[2, n-2]$. By definition, $P_{-}^{1} \subset P_{-}^{2} \subset \cdots \subset P^{n-1}$, so this induces a Morse decomposition of the flow [9].

The next example demonstrates that the criteria for $k$ positivity described in Thm. 1 are not invariant to coordinate transformations.

Example 1: Consider the symbolic matrix

$$
\bar{A}=\left[\begin{array}{cccc}
* & 0 & 0 & + \\
+ & * & + & 0 \\
0 & 0 & * & - \\
+ & 0 & - & *
\end{array}\right] .
$$

Since $\bar{A} \notin M_{2}^{4}$, the corresponding system is in general not even-positive. However, for the permutation matrix $P:=$ $\left[\begin{array}{llll}0 & 0 & 1 & 0 \\ 0 & 1 & 0 & 0 \\ 1 & 0 & 0 & 0 \\ 0 & 0 & 0 & 1\end{array}\right]$, we have

$$
\bar{B}:=P \bar{A} P^{\prime}=\left[\begin{array}{cccc}
* & 0 & 0 & - \\
+ & * & + & 0 \\
0 & 0 & * & + \\
- & 0 & + & *
\end{array}\right],
$$

so $\bar{B} \in M_{2}^{4}$. Thus, the system obtained by defining $y(t):=$ $P x(t)$ is even-positive.

The effect of certain coordinate transformations on the sign pattern of a matrix can be analyzed using a graphtheoretic approach.

\section{B. Influence graphs}

We associate with a sign matrix $\bar{A} \in\{*, 0,+,-\}^{n \times n}$ a signed and directed graph called the influence graph $G=$ $(V, E)$. The vertices are $V=\left\{x_{1}, \ldots, x_{n}\right\}$ (i.e., every vertex corresponds to a state-variable in the system $\dot{x}=\bar{A} x$ ). There is a directed edge from vertex $x_{j}$ to $x_{i}$ if $\bar{a}_{i j} \neq 0$ and $i \neq j$, and the sign of this edge is $\bar{a}_{i j}$. Note that there are no self-loops and that there it at most one edge from $x_{j}$ to $x_{i}$. There is a one-to-one correspondence between the influence graph of a system and its sign pattern, except for the entries on the diagonal of $\bar{A}$ (that are irrelevant for our considerations). Thus, with a slight abuse of notation we say that $\bar{A}$ satisfies some graph-theoretical property iff its influence graph satisfies this property.

The set of in-neighbors [out-neighbors] of a vertex $x_{i}$ is denoted by $\mathcal{N}_{\text {in }}\left(x_{i}\right)\left[\mathcal{N}_{\text {out }}\left(x_{i}\right)\right]$, and the set of neighbors of $x_{i}$ is $\mathcal{N}\left(x_{i}\right):=\mathcal{N}_{\text {in }}\left(x_{i}\right) \cup \mathcal{N}_{\text {out }}\left(x_{i}\right)$. The in-degree [outdegree $]$ of $x_{i}$ is $\left|\mathcal{N}_{\text {in }}\left(x_{i}\right)\right|\left[\left|\mathcal{N}_{\text {out }}\left(x_{i}\right)\right|\right]$, and the degree is $\left|\mathcal{N}\left(x_{i}\right)\right|$.
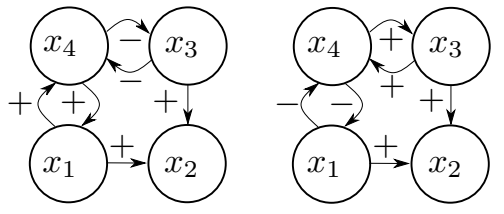

Fig. 1. Influence graphs corresponding to $\bar{A}$ (left) and $\bar{B}$ (right) in Example 1

For example, Fig. 11 depicts the influence graphs associated with the symbolic matrix $\bar{A}$ and $\bar{B}$ in Example 1

From here on we always assume that the influence graph is connected. Otherwise, we can simply treat each connected component separately.

\section{Main Results}

Consider a symbolic matrix $\bar{A}$ that is either odd-positive, even-positive, or both. Then the definition of the matrices $M_{k}^{n}$ implies that its associated influence graph satisfies the following properties:

(a) Degree constraint: $\left|\mathcal{N}\left(x_{i}\right)\right| \leq 2$ for any vertex $x_{i}$ in the graph.

(b) Sign-symmetric influence: for any two vertices $x_{i}, x_{j}$ in the graph, if the edge from $x_{i}$ to $x_{j}$ is $+[-]$ then there cannot be a $-[+]$ edge from $x_{j}$ to $x_{i}$.

Furthermore, if $\bar{A}$ is odd-positive then

(c) all edges are + ,

whereas if $\bar{A}$ is not odd-positive and is even-positive then

(d) there exist $r \in[1,2]$ edges that are - and all other edges are + .

\section{A. k-positivity up to permutations}

Our first two main results provide a necessary and sufficient graph-theoretic condition for $k$-positivity up to a permutation. Let $\mathbb{P} \subset \mathbb{R}^{n \times n}$ denote the set of permutation matrices.

Proposition 1: Let $\bar{A}$ be a symbolic matrix. The following two conditions are equivalent.

1) There exist an odd $k \in[2, n-2]$ and $P \in \mathbb{P}$ such that $P \bar{A} P^{\prime}$ is $k$-positive.

2) $\bar{A}$ satisfies properties (a) (b), and (c)

Proposition 2: Let $\bar{A}$ be a symbolic matrix. The following two conditions are equivalent.

(I) There exist an even $k \in[2, n-2]$ and $P \in \mathbb{P}$ such that $P \bar{A} P^{\prime}$ is $k$-positive, but there does not exist $P \in \mathbb{P}$ such that $P \bar{A} P^{\prime}$ is odd-positive.

(II) $\bar{A}$ satisfies properties (a) (b), and (d)

Example 2: The influence graph associated with the symbolic matrix $\bar{A}$ in (7) satisfies properties (a), (b) and (d)] so by Prop. 2 there exists $P \in \mathbb{P}$ such that $\overrightarrow{P A} P^{\prime}$ is $k$-positive. Furthermore, there does not exist a $P \in \mathbb{P}$ such that $P \bar{A} P^{\prime}$ is odd-positive.

Proof of Prop. 1] If 11 holds then the influence graph associated with $P \bar{A} P^{\prime}$ satisfies properties (a), (b), and (c)] Since a permutation amounts to relabeling the vertices, it is easy to see that this implies that the influence graph 
associated with $\bar{A}$ also satisfies properties (a), (b), and (c) We conclude that 10 implies 2).

To prove the converse implication, assume that 2 holds. If $\bar{A}$ is odd-positive then (1) holds for $P=I$. Thus, we may assume that $\bar{A}$ is not odd-positive. We consider several cases. Case 1. Suppose that every vertex has exactly two neighbors. Pick an arbitrary vertex, and associate with it a statevariable $y_{1}$. Pick one of its neighbors and associate with it the state-variable $y_{2}$. Now $y_{2}$ has a unique neighbor that is not $y_{1}$ and we denote it by $y_{3}$. We proceed in this way choosing at each step the only neighbor of the current vertex that has not been chosen yet. Since the influence graph is connected, this procedure ends after all vertices have been indexed in increasing order. The resulting influence graph is odd-positive. The $y_{i} \mathrm{~s}$ are clearly a permutation of the $x_{i} \mathrm{~s}$ i.e., $y=P x$, for some $P \in \mathbb{P}$.

Case 2. Suppose that every vertex has exactly two neighbors, except for two vertices, say $x_{i}$ and $x_{j}$, that have a single neighbor. Let $y_{1}:=x_{i}$ and $y_{n}:=x_{j}$. Since the graph is connected, these vertices cannot be neighbors. Denote the single neighbor of $y_{1}$ by $y_{2}$. Denote the single neighbor of $y_{2}$ that is not $y_{1}$ by $y_{3}$. We proceed in this way choosing at each step the only neighbor of the current vertex that has not been chosen yet. Since the influence graph is connected and all the vertices except for $y_{1}$ and $y_{n}$ have two neighbors, this procedure ends after all vertices have been indexed in increasing order. The resulting influence graph is oddpositive (and also even-positive).

Since we assume that 21 holds and the graph is connected, the two cases above are the only possible cases, and in each such case we showed that 10 holds. Thus, (2) implies 1 .

Proof of Prop. 2. Suppose that (I) holds. Then there exists $P \in \mathbb{P}$ such that $P \bar{A} P^{\prime}$ is even-positive, but not oddpositive. This implies that $P \bar{A} P^{\prime}$ satisfies properties (a) (b) and (d) Indeed, there must be at least one - edge in $P A P^{\prime}$, as otherwise it is also odd-positive. Since a permutation amounts to relabeling the vertices, $\bar{A}$ also satisfies properties (a), (b), and (d) We conclude that (I) implies (II).

To prove the converse implication, assume that (II) holds. If $\bar{A}$ is even-positive and not odd-positive then (I) holds for $P=I$. Thus, we may assume this is not so. We consider several cases.

Case 1. Suppose that all the edges are + except for two edges that are -, and that every vertex has exactly two neighbors. The sign-symmetric influence property implies that the edges are incident to two vertices, say, $x_{i}$ and $x_{j}$. Let $y_{1}:=$ $x_{i}$, and $y_{n}:=x_{j}$. Choose the only neighbor of $y_{1}$, that is not $y_{n}$, and associate with it the state-variable $y_{2}$. The rest of the construction is similar to that described in the proof of Prop. 1, with the exception that the resulting graph is even-positive and not odd-positive.

Case 2. Suppose that all the edges are + , except for a single edge that is - and that every vertex has exactly two neighbors. The sign-symmetric influence property implies that there exist vertices $x_{i}$ and $x_{j}$ such that the edge from $x_{i}$ to $x_{j}$ is - and there is no edge from $x_{j}$ to $x_{i}$. The argument for this case is very similar to the one in the previous case.
Case 3. Suppose that all the edges are + except for two edges that are - and that every vertex has exactly two neighbors except for two vertices, say, $x_{i}$ and $x_{j}$, that have a single neighbor. Since the graph is connected, $x_{i}$ and $x_{j}$ cannot be neighbors. Thus, the negative edges are incident to two vertices $x_{p}, x_{q}$, that are different than $x_{i}, x_{j}$. Let $y_{1}:=x_{p}$ and $y_{n}:=x_{q}$. Next, let $y_{2}$ be the neighbour of $y_{1}$ that is not $y_{n}$. We iterate over the vertices labeling them as $y_{3}, y_{4}$ and so on as done in the previous cases. This must end at either $x_{i}$ or $x_{j}$ as these are the only vertices with a single neighbor. W.1.o.g. assume that this is $x_{i}$. Now, let $y_{n-1}$ be the neighbour of $y_{n}$ that is not $y_{1}$. We iterate over the remaining vertices, labeling them as $y_{n-2}, y_{n-3}$ and so on until we end up with $x_{j}$, after which all the state variables have been relabeled. The resulting graph is even-positive, and not oddpositive.

Case 4. Suppose that all the edges are + , except for one edge that is - , and that every vertex has exactly two neighbors, except for two vertices that have a single neighbor. The proof for this case is very similar to the one in the previous case, except that now there is an edge from $x_{p}$ to $x_{q}$, but not from $x_{q}$ to $x_{p}$.

The four cases above are all the possible cases, and in each such case we showed that (I) holds.

Since the proofs of Props. 1 and 2 are constructive, they provide a way to actually determine a permutation that transforms $\bar{A}$ into a $k$-positive system. The next example demonstrates this.

Example 3: Consider again the matrix $\bar{A}$ in Example 1 This corresponds to Case 1 in the proof of Prop. 2. Following the construction in this case yields the relabeling

$$
y_{1}=x_{3}, y_{4}=x_{4}, y_{2}=x_{2}, y_{3}=x_{1},
$$

or

$$
y_{1}=x_{4}, y_{4}=x_{3}, y_{2}=x_{2}, y_{3}=x_{1} .
$$

The first of these is just the permutation used in Example 1 and applying this permutation indeed yields an even-positive system. It is easy to verify that this is true also for the permutation corresponding to the second relabeling.

The proofs of Props. 1 and 2 also provide the number of different permutations that yield a $k$-positive system.

Fact 1: Suppose that $\bar{A}$ is not $k$-positive for any $k \in$ $[2, n-2]$. If $\bar{A}$ satisfies properties (a), (b), and (d) then there exist 2 matrices in $\mathbb{P}$ such that $P A P^{\prime}$ is even-positive. If $\bar{A}$ satisfies properties (a) (b), and (c) then there exist $2 n$ matrices in $\mathbb{P}$ such that $P A P^{\prime}$ is odd-positive.

Proof: Suppose that $\bar{A}$ satisfies properties [a), (b)] and (d) After the relabeling the state-variables that correspond to vertices that are incident to negative edges must be the first and the last state-variables. Thus, there are two possible options to index them. After this choice is made, the indexing of all the other state-variables is predetermined as in the proof of Prop. 2 Indeed, any other choice yields a symbolic matrix $\bar{B}$ that has a non-zero entry $\bar{b}_{i j}$ with $1<$ $|i-j|<n-1$. Hence there are 2 possible permutations.

Now suppose that $\bar{A}$ satisfies properties (a) (b)] and (c) 
Due to symmetry, every state-variable can be chosen to be the first state-variable (i.e. $y_{1}$ ) yielding $n$ possibilities. After this choice is made, the indexing of all the other state-variables can be done in either increasing or decreasing order. Thus, there are overall $2 n$ possible permutations.

Next we study Problem 1 when we allow also scaling by a signature matrix (also called sign transformations). Note that if $S$ is a signature matrix then $S^{-1}=S$.

\section{B. k-positivity up to permutations and sign-transformations}

Using sign-transformations allows to detect more $k$ positive systems "in disguise". We begin with a simple example demonstrating this.

Example 4: Consider the symbolic matrix

$$
\bar{A}=\left[\begin{array}{cccc}
* & 0 & 0 & + \\
- & * & - & 0 \\
0 & 0 & * & - \\
+ & 0 & - & *
\end{array}\right] .
$$

The associated influence graph has four - edges, so Props. 1 and 2 imply that for any $k \in[2, n-2]$ there does not exist a $P \in \mathbb{P}$ so that $P \bar{A} P^{\prime}$ is $k$-positive. For the signature matrix $S=\operatorname{diag}\{1,-1,1,1\}$, the matrix

$$
S \bar{A} S=\left[\begin{array}{cccc}
* & 0 & 0 & + \\
+ & * & + & 0 \\
0 & 0 & * & - \\
+ & 0 & - & *
\end{array}\right],
$$

satisfies properties (a), (b), and (d) so Prop. 2 implies that there exists a permutation matrix $P$ such that $P S \bar{A} S P^{\prime}$ is even-positive.

Let $\mathbb{S} \subset \mathbb{R}^{n \times n}$ denote the set of signature matrices. We introduce the following definition.

Definition 2: We call the symbolic matrix $\bar{A}$ structurally odd-even-positive if there exist $P_{1} \in \mathbb{P}$ and $S_{1} \in \mathbb{S}$ such that $P_{1} S_{1} \bar{A} S_{1} P_{1}^{\prime}$ is odd-positive and there exist $P_{2} \in \mathbb{P}$ and $S_{2} \in \mathbb{S}$ so that $P_{2} S_{2} \bar{A} S_{2} P_{2}^{\prime}$ is even-positive. We call $\bar{A}$ structurally even-positive [structurally odd-positive] if it is not odd-even-positive, and there exist $P \in \mathbb{P}, S \in \mathbb{S}$ so that $P S \bar{A} S P^{\prime}$ is even-positive [odd-positive].

If $S \in \mathbb{S}$ then letting $y:=S x$ implies that either $y_{i}=x_{i}$ or $y_{i}=-x_{i}$ for all $i$. In the latter case, the effect on the influence graph is flipping the signs of all the edges incident to vertex $x_{i}$. This implies that properties (a) and (b) are invariant to sign-transformations. To study the effect of setting $y_{i}=-x_{i}$ on properties (c) and (d), we introduce more notation. For a symbolic matrix $\bar{A}$, let $\zeta(\bar{A})$ denote the number of pairs of neighbors $\left(x_{i}, x_{j}\right)$ such that there is an edge from $x_{i}$ to $x_{j}$ and/or from $x_{j}$ to $x_{i}$. For example, for the matrix $\bar{A}$ in $(8)$, we have that three such pairs: $\left(x_{1}, x_{2}\right)$, $\left(x_{2}, x_{3}\right)$, and $\left(x_{3}, x_{4}\right)$, so $\zeta(\bar{A})=3$.

Proposition 3: Let $\bar{A}$ be a symbolic matrix. The following two conditions are equivalent.

1) $\bar{A}$ is either structurally odd-even-positive or structurally odd-positive.

2) $\bar{A}$ satisfies properties (a) and (b) and $\zeta(\bar{A})$ is even.
Proposition 4: Let $\bar{A}$ be a symbolic matrix. The following two conditions are equivalent.

(I) $\bar{A}$ is structurally even-positive.

(II) $\bar{A}$ satisfies properties (a) and (b) and $\zeta(\bar{A})$ is odd.

Example 5: Consider the symbolic matrix $\bar{A}$ in Example 4 . It satisfies properties (a) (b) and $\zeta(\bar{A})=3$. Thus, Prop. 4 implies that $\bar{A}$ is structurally even-positive.

Proof of Prop. 3 . Suppose that 1 holds, i.e. there exist $P \in$ $\mathbb{P}$ and $S \in \mathbb{S}$ such that $\bar{B}:=P S \bar{A} S P^{\prime}$ is odd-positive. Then $\bar{B}$ satisfies properties (a) and (b) and thus so does $\bar{A}$. The matrix $\bar{B}$, and thus also the matrix $P^{\prime} \bar{B} P=S \bar{A} S$, satisfies property (c) This implies that $\zeta(S \bar{A} S)=0$. Since negation of a state-variable $x_{i}$ flips the signs of all the edges incident to the vertex $x_{i}, \zeta(\bar{A})$ must be even. Thus, 1 ) implies 2].

To prove the converse implication, assume that 2) holds. We consider two cases.

Case 1. If $\zeta(\bar{A})=0$ then all the edges are + , so $\bar{A}$ satisfies properties (a), (b), and (d), and combining this with Prop. 1 implies that there exists $P \in \mathbb{P}$ such that $P \bar{A} P^{\prime}$ is oddpositive.

Case 2. If $\zeta(\bar{A})>0$ then pick a vertex $x_{i}$ that is incident to a - edge and apply a negation on this vertex. If $x_{i}$ was connected to all its neighbors (where the number of neighbors is either one or two) by - edges then after the negation it is connected to all of them by + edges. Thus, $\zeta\left(S_{i} \bar{A} S_{i}\right)=\zeta(\bar{A})-2$, where $S_{i} \in \mathbb{S}$ corresponds to the negation of $x_{i}$. If $\zeta\left(S_{i} \bar{A} S_{i}\right)=0$ then we conclude as in Case 1 that there exists $P \in \mathbb{P}$ such that $P S_{i} \bar{A} S_{i} P^{\prime}$ is odd-positive. Else, we pick another vertex that is incident to a negative edge and continue the process.

If $x_{i}$ was connected to one neighbor $x_{j}$ by an - edge and to another neighbor $x_{q}$ by an + edge then after the negation it is connected to $x_{q}$ by an - edge. We proceed by negating $x_{q}$. We continue this procedure until $\zeta$ decreases by 2 . This is bound to eventually take place, since every time we apply a negation neighbor after neighbor, we "push" the negative edges in the same "direction", until we finally apply a negation on a vertex that is connected to all its neighbors by negative edges (recall that $\zeta(\bar{A})$ is positive and even).

Then either there are no more negative edges in the graph, or we repeat the process again. Since it is clear that we do not apply a negation on any state variable more than once, this process is finite and terminates after decreasing $\zeta$ to zero. We conclude as in Case 1 that that there exists $P \in \mathbb{P}$ such that $P S \bar{A} S P^{\prime}$ is odd-positive, where $S \in \mathbb{S}$ represents all the negations used in the process.

Thus, we showed that 2) implies (1).

Proof of Prop.4. Suppose that (I) holds, i.e. there exist $P \in$ $\mathbb{P}$ and $S \in \mathbb{S}$ such that $\bar{B}:=P S \bar{A} S P^{\prime}$ is even-positive, yet $\bar{A}$ is not structurally odd-even positive. Then $\bar{B}$ satisfies properties (a) and (b), and thus so does $\bar{A}$. The matrix $\bar{B}$, and thus also the matrix $P^{\prime} \bar{B} P=S \bar{A} S$, satisfies property (d). Combining this with the fact that $\bar{A}$ is not structurally oddeven positive implies that $\zeta(S \bar{A} S)=1$. Since negation of a state-variable $x_{i}$ flips the signs of all the edges incident to the vertex $x_{i}, \zeta(\bar{A})$ must be odd. Thus, (I) implies (II) 
To prove the converse implication, assume that (II) holds. Arguing as in the proof of Prop. 3, we can find an $S \in \mathbb{S}$ such that $\zeta(S \bar{A} S)=1$. This implies that $r$, the number of - edges, satisfies $r \in\{1,2\}$. Thus, $S \bar{A} S$ satisfies properties (a) (b) and (d) Applying Prop. 2 completes the proof of Prop. 4

Remark 1: Since the proof of Props. 3 and 4 are constructive, they provide an algorithm for finding the transformations that yield a $k$-positive system.

\section{AN APPLICATION}

Consider the nonlinear Lotka-Volterra system:

$$
\dot{x}_{i}=x_{i}\left(r_{i}+\sum_{j=1}^{n} a_{i j} x_{j}\right), \quad i \in[1, n],
$$

with $r_{i}, a_{i j} \in \mathbb{R}$. Such systems play an important role in mathematical ecology [11]. The relevant state-space in these applications is $\mathbb{R}_{+}^{n}$.

Proposition 5: Consider (9) with $n \geq 4$ and such that:

1) for any $i \in[1, n]$ there are at most two values $j \neq i$ such that $a_{i j}$ is non-zero;

2) $a_{i j} a_{j i} \geq 0$ for any $i, j$.

Then there exist $P \in \mathbb{P}, S \in \mathbb{S}$ such that the system obtained by setting $y(t):=P S x(t)$ is $k$-cooperative for some $k \in$ $[2, n-2]$.

Proof: Let $A:=\left\{a_{i j}\right\}_{i, j=1}^{n}$. For any $i \neq j$, the $(i, j)$ entry in the Jacobian of (9) is $J_{i j}(x)=a_{i j} x_{i}$. This implies that, ignoring diagonal entries (that are not relevant for our purposes), $J(x)$ has the same sign pattern as $A$ for all $x \in$ $\mathbb{R}_{+}^{n}$. The influence graph of $A$ satisfies properties (a) and (b) Combining this with Props. 3 and 4 completes the proof.

Note that the fact that the nonlinear $y$-system is $k$ cooperative has important implications. If $k$ is odd then the system is 1-cooperative, i.e. cooperative. If $k$ is even then the system is 2-cooperative and, under an additional irreducibility assumption, it satisfies the strong PoincaréBendixson property described in [25]: any omega limit set of a bounded trajectory that does not include an equilibrium is a periodic orbit.

\section{CONCLUSION}

$k$-positive systems generalize the well-known positive systems. An important advantage of such systems is the existence of simple sign-pattern conditions guaranteeing that a system is $k$-positive. It was recently shown that $k$-positive systems provide a useful tool for analyzing the asymptotic behavior of nonlinear systems. However, $k$-positivity is not invariant under coordinate transformations.

Here, we derived graph-theoretic necessary and sufficient conditions for a system to be $k$-positive up to two types of transformations: permutations of the state-variables and scaling by a signature matrix. We also provided algorithms that explicitly find such transformations, when they exist.

It would be interesting to extend these results to more general coordinate transformations.

\section{REFERENCES}

[1] R. Al-Seidi, M. Margaliot, and J. Garloff, "Discrete-time $k$-positive linear systems," IEEE Trans. Automat. Control, 2019, to appear. [Online]. Available: https://arxiv.org/abs/1910.08125

[2] R. Alseidi, M. Margaliot, and J. Garloff, "On the spectral properties of nonsingular matrices that are strictly sign-regular for some order with applications to totally positive discrete-time systems," J. Math. Anal. Appl., vol. 474, pp. 524-543, 2019.

[3] Z. Aminzare and E. D. Sontag, "Contraction methods for nonlinear systems: A brief introduction and some open problems," in Proc. 53rd IEEE Conf. on Decision and Control, Los Angeles, CA, 2014, pp. 3835-3847.

[4] D. Angeli and E. D. Sontag, "Monotone control systems," IEEE Trans. Automat. Control, vol. 48, pp. 1684-1698, 2003.

[5] M. Arcak and E. Sontag, "Diagonal stability of a class of cyclic systems and its connection with the secant criterion," Automatica, vol. 42, pp. 1531-1537, 2006.

[6] E. Bar-Shalom, A. Ovseevich, and M. Margaliot, "Ribosome flow model with different site sizes," SIAM J. Applied Dynamical Systems, vol. 19, no. 1, pp. 541-576, 2020.

[7] A. Berman and R. J. Plemmons, Nonnegative Matrices in the Mathematical Sciences. SIAM, 1987.

[8] L. Farina and S. Rinaldi, Positive Linear Systems: Theory and Applications. John Wiley, 2000.

[9] T. Gedeon and G. Hines, "Multi-valued characteristics and Morse decompositions," J. Diff. Eqns., vol. 247, no. 4, pp. 1013-1042, 2009.

[10] G. Haag, Modelling with the Master Equation: Solution Methods and Applications in Social and Natural Sciences. Springer International Publishing, 2017.

[11] J. Hofbauer and K. Sigmund, The Theory of Evolution and Dynamical Systems: Mathematical Aspects of Selection. Cambridge University Press, 1988

[12] E. Kaszkurewicz and A. Bhaya, Matrix Diagonal Stability in Systems and Computation. Boston, MA: Birkhauser, 2000.

[13] R. Katz, M. Margaliot, and E. Fridman, "Entrainment to subharmonic solutions in oscillatory discrete-time systems," Automatica, 2019, to appear. [Online]. Available: https://arxiv.org/abs/1904.06547

[14] A. S. Landsberg and E. J. Friedman, "Dynamical effects of partial orderings in physical systems," Phys. Rev. E, vol. 54, pp. 3135-3141, 1996.

[15] J. Mallet-Paret and H. L. Smith, "The Poincaré-Bendixson theorem for monotone cyclic feedback systems," J. Dyn. Differ. Equ., vol. 2, no. 4, pp. 367-421, 1990.

[16] M. Margaliot and E. D. Sontag, "Revisiting totally positive differential systems: A tutorial and new results," Automatica, vol. 101, pp. 1-14, 2019.

[17] A. Rantzer and M. E. Valcher, "A tutorial on positive systems and large scale control," in Proc. 57th IEEE Conf. on Decision and Control, Miami Beach, FL, USA, 2018, pp. 3686-3697.

[18] A. Rantzer, "Scalable control of positive systems," European J. Control, vol. 24, pp. 72-80, 2015.

[19] I. W. Sandberg, "On the mathematical foundations of compartmental analysis in biology, medicine, and ecology," IEEE Trans. Circuits and Systems, vol. 25, no. 5, pp. 273-279, 1978.

[20] B. Schwarz, "Totally positive differential systems," Pacific J. Math., vol. 32, no. 1, pp. 203-229, 1970 .

[21] J. Smillie, "Competitive and cooperative tridiagonal systems of differential equations," SIAM J. Math. Anal., vol. 15, pp. 530-534, 1984.

[22] H. L. Smith, "Periodic tridiagonal competitive and cooperative systems of differential equations," SIAM J. Math. Anal., vol. 22, no. 4, pp. 1102-1109, 1991.

[23] H. L. Smith, Monotone Dynamical Systems: An Introduction to the Theory of Competitive and Cooperative Systems, ser. Mathematical Surveys and Monographs. Providence, RI: Amer. Math. Soc., 1995, vol. 41.

[24] H. L. Smith, "Is my system of ODEs cooperative?" 2012. [Online]. Available: https://math.la.asu.edu/ halsmith/identifyMDS.pdf

[25] E. Weiss and M. Margaliot, "A generalization of linear positive systems with applications to nonlinear systems: Invariant sets and the Poincaré-Bendixson property," 2019, submitted. [Online]. Available: https://arxiv.org/abs/1902.01630

[26] Y. Zarai, M. Margaliot, and T. Tuller, "Modeling and analyzing the flow of molecular machines in gene expression," in Systems Biology, N. Rajewsky, S. Jurga, and J. Barciszewski, Eds. Springer, 2018, pp. 275-300. 